\title{
Theory of nuclear excitation by electron capture for heavy ions
}

\author{
Adriana Pálffy and Werner Scheid \\ Institut für Theoretische Physik, Justus-Liebig-Universität Giessen, \\ Heinrich-Buff-Ring 16, 35392 Giessen, Germany* \\ Zoltán Harman \\ Max-Planck-Institut für Kernphysik, \\ Saupfercheckweg 1, 69117 Heidelberg, Germany
}

(Dated: October 29, 2018)

\begin{abstract}
We investigate the resonant process of nuclear excitation by electron capture, in which a continuum electron is captured into a bound state of an ion with the simultaneous excitation of the nucleus. In order to derive the cross section a Feshbach projection operator formalism is introduced. Nuclear states and transitions are described by a nuclear collective model and making use of experimental data. Transition rates and total cross sections for NEEC followed by the radiative decay of the excited nucleus are calculated for various heavy ion collision systems.
\end{abstract}

PACS numbers: 34.80.Lx, 23.20.Nx, 23.20.-g

Keywords: electron recombination, nuclear excitation, resonant transitions, highly charged ions

*Electronic address: Adriana-Claudia.Gagyi-Palffy@uni-giessen.de 


\section{INTRODUCTION}

In the resonant process of nuclear excitation by electron capture (NEEC) a free electron is captured into a bound atomic shell with the simultaneous excitation of the nucleus. It is the nuclear physics analogue of dielectronic recombination (DR), where a resonant excitation of a bound electron occurs. NEEC is the time-reversed process of internal conversion (IC). The excited nucleus can then decay radiatively or by internal conversion. In the later case, a resonant inelastic electron scattering on the nucleus occurs.

The NEEC recombination mechanism has been presented for the first time in Ref. [1]. Several studies have been made concerning NEEC in plasmas [1, 2] or in solid targets [3, 4, 5]. In [3], cross sections of the process are calculated through a scaling procedure applied to the results of DR, considering that the two processes differ only in their excitation mechanisms. Following that, in Ref. [4], similar estimates of NEEC cross sections are obtained for the same nuclei by using experimental nuclear rather than atomic data. A more theoretical approach is provided in Ref. [2] through an explicit treatment of the electron-nucleus interaction in the capture process, following the theory used for the calculation of IC coefficients from Ref. [6]. In Ref. [5], non-relativistic calculations are presented for the case of NEEC into bare ions channeling through single crystals. The results are in disagreement with the previous ones from [3, 4]. As NEEC has not been observed experimentally yet, neither in plasmas nor in the case of ions in crystals or colliding with electron targets, the magnitude of its cross section is in doubt. Similar discrepancies exist also in the case of theoretical calculations for the similar process of nuclear excitation by electron transition (NEET). NEET is a fundamental but rare mode of decay of an excited atomic state in which the energy of atomic excitation is transferred to the nucleus. This corresponds to the time-reversed bound state internal conversion. Unlike NEEC, NEET has been observed experimentally [7], in the same year in which direct evidence of the bound internal conversion [8] has been reported, thus opening a new period in which experimental precision allows atomic shell models to have regard for the internal structure of the nucleus. The permanent development of the experimental techniques and the enhanced possibilities of preparing bare ions and electron targets make the experimental observation of NEEC a reasonable goal for the foreseeable future. Thus theoretical calculations for NEEC occurring in scattering measurements are particularly useful, especially in finding candidate isotopes and transitions suitable for experimental 
observation.

In both NEEC and NEET, that are at the border-line between atomic and nuclear physics, electronic orbital energy is converted directly into nuclear energy. They offer therefore the possibility to explore the spectral properties of heavy nuclei through atomic physics experiments. Experimental techniques developed for scattering studies of electron recombination with atomic ions, e.g. experiments with stored [9, 10] or trapped [11, 12] ions, can be applied to gain information on the nuclear structure of several nuclides which is hardly accessible by nuclear scattering experiments. Especially, NEEC is expected to allow the determination of nuclear transition energies, the study of atomic vacancy effects on nuclear lifetime and population mechanisms of excited nuclear levels.

In this work we calculate total cross sections for NEEC followed by the radiative nuclear decay for various transitions in heavy elements. Particular interest has been payed for collision systems where experimental requirements for the observation of NEEC are likely to be fulfilled. In order to derive the cross section formula for the process we extended the Feshbach projector formalism developed and used for the DR [13] to account for the interaction of electronic and nuclear degrees of freedom. The electric and magnetic electronnucleus interactions are considered explicitly and the nucleus is described with the help of a nuclear collective model [14]. The dynamics of electrons is governed by the Dirac equation as required in the case of high-Z elements. This formalism is presented in Section III. The calculation of the NEEC rates for the electric and magnetic transitions as well as the nuclear model are described in Section III. Numerical results of the calculation are given in Section [V] We conclude with a short Summary (Section V). The derivation of the magnetic interaction operator related to Section III, as well as some larger formulas involved in Section III are given in the Appendix. In this work atomic units have been used unless otherwise mentioned.

\section{THEORY OF NUCLEAR EXCITATION BY ELECTRON CAPTURE}

In this section we derive the total cross section formula for the NEEC process followed by the radiative decay of the excited nucleus. We consider that the electron is captured into the bound state in the Coulomb field of a bare nucleus and that only the nucleus is decaying by emitting a photon. However, the derivation of the cross section can be extended to the 
many-electron case in a straightforward way and also an additional radiative decay of the electron can be treated by applying the formalism.

\section{A. Decomposition of the Fock space by means of projection operators}

The initial state $\left|\Psi_{i}\right\rangle$ of the system consisting of the nucleus in its ground state, the free electron, and the vacuum state of the electromagnetic field can be written as a direct product of the nuclear, electronic, and photonic state vectors:

$$
\left|\Psi_{i}\right\rangle=\left|N, \vec{p} m_{s}, 0\right\rangle \equiv|N\rangle \otimes\left|\vec{p} m_{s}\right\rangle \otimes|0\rangle
$$

Here, $\vec{p}$ is the asymptotic momentum of the electron, $m_{s}$ its spin projection, and $|N\rangle$ the nuclear ground state. The state $\left|\Psi_{d}\right\rangle$ formed by the resonant capture has the form

$$
\left|\Psi_{d}\right\rangle=\left|N^{*}, n_{d} \kappa_{d} m_{d}, 0\right\rangle \equiv\left|N^{*}\right\rangle \otimes\left|n_{d} \kappa_{d} m_{d}\right\rangle \otimes|0\rangle
$$

with $n_{d}, \kappa_{d}$, and $m_{d}$ being the principal quantum number, Dirac angular momentum, and magnetic quantum numbers of the bound one-electron state, respectively. The excited nuclear state is denoted by $\left|N^{*}\right\rangle$. The final state $\left|\Psi_{f}\right\rangle$ of the NEEC process contains a photon with the wave number $\vec{k}$ and the transversal polarization $\sigma=1,2$, and the nucleus which is again in its ground state $|N\rangle$ :

$$
\begin{gathered}
\left|\Psi_{f}\right\rangle=\left|N, n_{d} \kappa_{d} m_{d}, \vec{k} \sigma\right\rangle \equiv|N\rangle \otimes\left|n_{d} \kappa_{d} m_{d}\right\rangle \otimes|\vec{k} \sigma\rangle \\
|\vec{k} \sigma\rangle=a_{\vec{k} \sigma}^{\dagger}|0\rangle
\end{gathered}
$$

Here, the $a_{\vec{k} \sigma}^{\dagger}$ is a photon creation operator. The corresponding conjugate annihilation operator is denoted by $a_{\vec{k} \sigma}$.

To clearly separate these states in the perturbative expansion of the transition operator, we introduce operators projecting onto the individual subspaces. Characterizing the state of the electron in the positive part of the continuous spectrum by the energy $\varepsilon$ rather than the momentum of the free electron, we write the projector $P$ belonging to the first type of subspace as

$$
P=\int d \varepsilon \sum_{\alpha}|\alpha \varepsilon\rangle\langle\alpha \varepsilon|
$$


For brevity we introduce the multi-index $\alpha$ to stand for all discrete quantum numbers of the total system. The projection operator of the subspace spanned by states of the type (2) is written as

$$
Q=\sum_{q}|q\rangle\langle q|,
$$

with the cumulative index $q$ introduced again to summarize all discrete quantum numbers describing the bound electron and the excited nucleus. The subspace of the state vectors containing one transverse photon is associated with the projection operator

$$
R=\sum_{q} \sum_{\vec{k} \sigma} a_{\vec{k} \sigma}^{\dagger}|q\rangle\langle q| a_{\vec{k} \sigma} .
$$

Assuming corrections due to two- or more-photon states [15] and due to the presence of the negative electronic continuum to be negligible, we postulate the following completeness relation:

$$
P+Q+R=\mathbf{1}
$$

where $\mathbf{1}$ is the unity operator of the Fock space.

\section{B. The total Hamiltonian of the system}

The total Hamiltonian operator for the system consisting of the nucleus, the electron, and the radiation field can be written as

$$
H=H_{n}+H_{e}+H_{r}+H_{e n}+H_{e r}+H_{n r} .
$$

The Hamiltonian of the nucleus $H_{n}$ is written in terms of the nuclear collective model [14] by using creation and annihilation operators of the collective modes, $B_{\lambda \mu}^{\dagger}$ and $B_{\lambda \mu}$,

$$
H_{n}=\sum_{\lambda \mu} \Omega_{\lambda} B_{\lambda \mu}^{\dagger} B_{\lambda \mu}
$$

where $\Omega_{\lambda}$ are the phonon frequencies. The Dirac Hamiltonian of the free electron is given by

$$
H_{e}=c \vec{\alpha} \cdot \vec{p}+(\beta-1) c^{2},
$$

whilst the pure quantized radiation field is described by

$$
H_{r}=\sum_{\vec{k} \sigma} \omega_{k} a_{\vec{k} \sigma}^{\dagger} a_{\vec{k} \sigma} .
$$


Interactions between the three subsystems are described by the three remaining Hamiltonians in eq. (91). We adopt the Coulomb gauge for the electron-nucleus interaction because it allows the separation of the dominant Coulomb attraction between the electronic and the nuclear degrees of freedom,

$$
H_{e n}=\int d^{3} r_{n} \frac{\rho_{n}\left(\vec{r}_{n}\right)}{\left|\vec{r}_{e}-\vec{r}_{n}\right|} .
$$

In eq. (13), $\rho_{n}\left(\vec{r}_{n}\right)$ is the nuclear charge density and the integration is performed over the whole nuclear volume. The interaction of the electron with the transverse photon field quantized in unit volume is given by

$$
H_{e r}=\sum_{\vec{k} \sigma} \sqrt{\frac{2 \pi c^{2}}{\omega_{k}}} \vec{\alpha}\left(\vec{\epsilon}_{\vec{k} \sigma} e^{i \vec{k} \cdot \vec{r}_{e}} a_{\vec{k} \sigma}+\text { h.c. }\right) .
$$

Here, $\vec{\alpha}$ is the vector of the Dirac $\alpha$ matrices $\left(\alpha_{x}, \alpha_{y}, \alpha_{z}\right)$, and $\vec{\epsilon}_{\vec{k} \sigma}$ is the polarization vector of the photons. Similarly, the interaction of the nucleus with the electromagnetic field is given by the Hamiltonian

$$
H_{n r}=-\sum_{\vec{k} \sigma} \int d^{3} r_{n} \vec{j}_{n}\left(\vec{r}_{n}\right) \sqrt{\frac{2 \pi}{\omega_{k}}}\left(\vec{\epsilon}_{\vec{k} \sigma} e^{i \vec{k} \cdot \vec{r}_{n}} a_{\vec{k} \sigma}+\text { h.c. }\right),
$$

where $\vec{j}_{n}\left(\vec{r}_{n}\right)$ is the nuclear current.

Using the projection operators we can separate the Hamiltonian as follows:

$$
H=H_{0}+V
$$

with

$$
\begin{gathered}
H_{0}=P H P+Q H Q+R H R \\
V \equiv H-H_{0}=P H Q+Q H P+P H R+R H P \\
+R H Q+Q H R .
\end{gathered}
$$

In this way the effect of the nuclear potential on bound and continuum electron states is included to all orders. The individual terms in the perturbation operator describe transitions between the different subspaces. For example, $P H Q$ describes the transition of the bound electron to the continuum, i.e. IC, and $Q H P$ accounts for the time-reversed process of IC, namely, NEEC. $P H R$ and $R H P$ are the lowest-order operators for photoionization and radiative recombination, respectively. $Q H R$ and $R H Q$ account for the radiative excitation of the nucleus and radiative decay of the nucleus or of the electron in a bound state. 


\section{Perturbation expansion of the transition operator}

The transition operator is defined as

$$
T(z)=V+V G(z) V
$$

where $G(z)$ is the Green operator of the system given by

$$
G(z)=(z-H)^{-1} .
$$

Here, $z$ is a complex energy variable. The cross section for a process can be expressed by the transition operator as follows:

$$
\frac{d \sigma_{i \rightarrow f}}{d \Omega_{k}}(E)=\frac{2 \pi}{F_{i}} \lim _{\epsilon \rightarrow 0+}\left|\left\langle\Psi_{f}|T(E+i \epsilon)| \Psi_{i}\right\rangle\right|^{2} \rho_{f},
$$

with the $\Psi_{f}$ and $\Psi_{i}$ as final and initial eigenstates of $H_{0}$, respectively. This cross section is differential with respect to the angle $\Omega_{k}$ of the photon emitted in the process. $F_{i}$ denotes the flux of the incoming electrons, and $\rho_{f}$ the density of the final photonic states.

We use the Lippmann-Schwinger equation to write the perturbation series for $T(z)$ in powers of $V$ with the Green function $G_{0}(z)$ of the unperturbed Hamiltonian $H_{0}$ :

$$
T(z)=V+V G_{0}(z) V+V G_{0}(z) V G_{0}(z) V+\ldots .
$$

Since the initial state of the NEEC process is by definition an eigenstate of $P$, and the final state is an eigenstate of $R$, we consider the projection $R T P$ of the transition operator:

$$
\begin{aligned}
R T P=R V P & +R V G_{0} V P+R V G_{0} V G_{0} V P \\
& +R V G_{0} V G_{0} V G_{0} V P+\ldots
\end{aligned}
$$

Here and in the following we omit the argument $z$. The first term in eq. (23) does not contribute to the NEEC process. After a further analysis of the second term $R V G_{0} V P=$ $R H_{n r} Q G_{0} Q H_{e n} P$ and inserting the spectral resolution (6) of $Q$ in the second order in $V$ we arrive to

$$
\left\langle\Psi_{f}|R T P| \Psi_{i}\right\rangle=\sum_{q} \frac{\left\langle N, n_{d} \kappa_{d} m_{d}, \vec{k} \sigma\left|H_{n r}\right| q\right\rangle\left\langle q\left|H_{e n}\right| N, \vec{p} m_{s}, 0\right\rangle}{z-E_{q}^{0}} .
$$

The energy $E_{q}^{0}$ denotes the unperturbed eigenvalue of the state $|q\rangle$. If we continue analyzing the perturbation expansion (23), the term of third order in $V$ can be written as

$$
\begin{aligned}
R V G_{0} V G_{0} V P= & R H_{e r} P G_{0} P H_{e n} Q G_{0} Q H_{e n} P \\
& +R H_{e r} P G_{0} P H_{e r} R G_{0} R H_{e r} P \\
& +R H Q G_{0} Q H R G_{0} R H_{e r} P .
\end{aligned}
$$


The first two terms do not contribute to the cross section of the considered NEEC process. The last term is decomposed as

$$
\begin{aligned}
R H Q G_{0} Q H R G_{0} R H_{e r} P= & R H_{e r} Q G_{0} Q H_{e r} R G_{0} R H_{e r} P \\
& +R H_{e r} Q G_{0} Q H_{n r} R G_{0} R H_{e r} P \\
& +R H_{n r} Q G_{0} Q H_{e r} R G_{0} R H_{e r} P \\
& +R H_{n r} Q G_{0} Q H_{n r} R G_{0} R H_{e r} P .
\end{aligned}
$$

Here, the first two terms are not considered, as they describe recombination by radiative decay of the electron. The process incorporated in the third term of (26) is not possible. The remaining last term, $Q H_{n r} R G_{0} R H_{e r} P$, accounts for the capture of the free electron by exchanging a virtual transverse photon with the nucleus. As we show in Appendix A, it can be approximated by $Q H_{\text {magn }} P$, where

$$
H_{\text {magn }}=-\frac{1}{c} \vec{\alpha} \int d^{3} r_{n} \frac{\vec{j}_{n}\left(\vec{r}_{n}\right)}{\left|\vec{r}-\vec{r}_{n}\right|}=-\vec{\alpha} \cdot \vec{A}(\vec{r})
$$

is the magnetic interaction Hamiltonian.

We continue the expansion (23) of the $T$ operator and only consider the terms that contain $Q H_{e n} P$ as the first step and $R H_{n r} Q$ as the final step. The contribution of order $V^{4}$ can be decomposed as

$$
\begin{aligned}
& R V G_{0} V G_{0} V G_{0} V P=R H_{n r} Q G_{0} Q H_{n e} P G_{0} P H_{n e} Q G_{0} Q H_{n e} P \\
& +R H_{n r} Q G_{0} Q\left(H_{e r}+H_{n r}\right) R G_{0} R\left(H_{e r}+H_{n r}\right) Q G_{0} Q H_{n e} P .
\end{aligned}
$$

We rewrite the first term as

$$
\begin{aligned}
& R H_{n r} Q G_{0} Q H_{n e} P G_{0} P H_{n e} Q G_{0} Q H_{n e} P= \\
& \sum_{q, q^{\prime}} R H_{n r} Q G_{0}|q\rangle\left\langle q\left|H_{n e} P G_{0} P H_{n e}\right| q^{\prime}\right\rangle\left\langle q^{\prime}\right| G_{0} Q H_{n e} P,
\end{aligned}
$$

and consider the diagonal matrix element

$$
\left\langle q\left|H_{n e} P G_{0} P H_{n e}\right| q\right\rangle=\int d \varepsilon \sum_{\alpha} \frac{\left\langle q\left|H_{n e}\right| \alpha \varepsilon\right\rangle\left\langle\alpha \varepsilon\left|H_{n e}\right| q\right\rangle}{z-E^{0}},
$$

with $E^{0}$ defined by $H_{0}\left|\alpha E^{0}\right\rangle=E^{0}\left|\alpha E^{0}\right\rangle$. Using the equality

$$
\lim _{\epsilon \rightarrow 0_{+}} \frac{1}{x+i \epsilon}=\mathcal{P}\left(\frac{1}{x}\right)-i \pi \delta(x),
$$


it can be further decomposed into

$$
\begin{gathered}
\int d \varepsilon \sum_{\alpha} \frac{\left\langle q\left|H_{n e}\right| \alpha \varepsilon\right\rangle\left\langle\alpha \varepsilon\left|H_{n e}\right| q\right\rangle}{z-E^{0}}=\Delta E_{q}^{\mathrm{NP}}-\frac{i}{2} \Gamma_{q}^{\mathrm{IC}} \\
\Delta E_{q}^{\mathrm{NP}} \equiv \mathcal{P} \int d \varepsilon \sum_{\alpha} \frac{\left\langle q\left|H_{n e}\right| \alpha \varepsilon\right\rangle\left\langle\alpha \varepsilon\left|H_{n e}\right| q\right\rangle}{z-E^{0}} \\
\Gamma_{q}^{\mathrm{IC}} \equiv i \pi \sum_{\alpha}\left|\left\langle q\left|H_{n e}\right| \alpha E^{0}\right\rangle\right|^{2}
\end{gathered}
$$

The notation $\Delta E_{q}^{\mathrm{NP}}$ was introduced to denote the Coulomb nuclear polarization correction to the energy of the state $q$ and $\Gamma_{q}^{\mathrm{IC}}$ for its internal conversion width. $\mathcal{P}$ denotes the principal value of the integral.

In a similar manner, the second term of (28) can be analyzed. It can be separated into the following four parts:

$$
\begin{aligned}
R H_{n r} Q G_{0} Q\left(H_{e r}+\right. & \left.H_{n r}\right) R G_{0} R\left(H_{e r}+H_{n r}\right) Q G_{0} Q H_{n e} P= \\
& R H_{n r} Q G_{0} Q H_{e r} R G_{0} R H_{e r} Q G_{0} Q H_{n e} P \\
& +R H_{n r} Q G_{0} Q H_{n r} R G_{0} R H_{n r} Q G_{0} Q H_{n e} P \\
& +R H_{n r} Q G_{0} Q H_{e r} R G_{0} R H_{n r} Q G_{0} Q H_{n e} P \\
& +R H_{n r} Q G_{0} Q H_{n r} R G_{0} R H_{e r} Q G_{0} Q H_{n e} P .
\end{aligned}
$$

The first term describes the emission and reabsorption of a photon by the electron recombined into the bound state. Its diagonal matrix element possesses a real and an imaginary part:

$$
\left\langle q\left|H_{e r} R G_{0} R H_{e r}\right| q\right\rangle=\Delta E_{q}^{\mathrm{SE}}-\frac{i}{2} \Gamma_{q}^{\mathrm{e}, \mathrm{rad}} .
$$

$\Delta E_{q}^{\mathrm{SE}}$ describes the one-loop self energy correction to the bound state energy of the electron. The imaginary part is the radiative decay rate of the electronic state and vanishes in the case of electron capture into the ground state of the ion. The second term in (35) describes the emission and a subsequent reabsorption of a virtual photon by the nucleus, and its diagonal matrix element reads

$$
\left\langle q\left|H_{n r} R G_{0} R H_{n r}\right| q\right\rangle=\Delta E_{q}^{\mathrm{NSE}}-\frac{i}{2} \Gamma_{q}^{\mathrm{n}, \mathrm{rad}}
$$

Here, $\Delta E_{q}^{\mathrm{NSE}}$ is the nuclear self energy correction to the energy of the ion, and $\Gamma_{q}^{\mathrm{n}, \mathrm{rad}}$ stands for the radiative decay width of the nucleus in the state $q$. The last two terms of (35) 
incorporate corrections to the intermediate state energy due to the exchange of a virtual transverse photon between the electronic and the nuclear currents. These corrections are neglected in our treatment as they are expected to be far less than the overall accuracy of experimental nuclear excitation energies.

Continuing the expansion (23) of the $T$ operator, the matrix element of the intermediate state Green operator in fourth order contains terms of the form

$$
\left\langle q\left|H_{i} G_{0} H_{i} G_{0} H_{i} G_{0} H_{i}\right| q\right\rangle=\sum_{q^{\prime}} \frac{\left\langle q\left|H_{i} G_{0} H_{i}\right| q^{\prime}\right\rangle\left\langle q^{\prime}\left|H_{i} G_{0} H_{i}\right| q\right\rangle}{z-E_{q^{\prime}}},
$$

where the label $i$ stands for $n e$, er and $n r$. We adopt the so-called isolated resonances approximation by taking only the diagonal matrix elements into account, i.e. we set $q=q^{\prime}$ in (38). This approximation is valid if the distance between neighboring resonances is large with respect to their total natural widths, which is the case in all systems we studied. Higher-order terms can be summed then as a geometric progression

$$
\frac{1}{z-E_{q}^{0}} \sum_{k=0}^{\infty} x^{k}=\frac{1}{z-E_{q}^{0}} \frac{1}{1-x}
$$

with the dimensionless quotient

$$
\begin{aligned}
x=\frac{1}{z-E_{q}^{0}} & \left(\left\langle q\left|H_{n e} P G_{0} P H_{n e}\right| q\right\rangle\right. \\
& \left.+\left\langle q\left|H_{e r} R G_{0} R H_{e r}\right| q\right\rangle+\left\langle q\left|H_{n r} R G_{0} R H_{n r}\right| q\right\rangle\right),
\end{aligned}
$$

resulting in

$$
\frac{1}{z-E_{q}^{0}-\Delta E_{q}^{\mathrm{NP}}-\Delta E_{q}^{\mathrm{SE}}-\Delta E_{q}^{\mathrm{NSE}}+\frac{i}{2} \Gamma_{q}^{\mathrm{IC}}+\frac{i}{2} \Gamma_{q}^{\mathrm{n}, \mathrm{rad}}} .
$$

Thus, the infinite perturbation expansion introduces energy corrections and widths into the energy denominator of the lowest order amplitude (24). The final expression for the transition amplitude of NEEC into states denoted by $d$ and followed by radiative nuclear decay is then

$$
\begin{aligned}
& \left\langle\Psi_{f}|R T(z) P| \Psi_{i}\right\rangle= \\
& \sum_{d} \frac{\left\langle N, n_{d} \kappa_{d} m_{d}, \vec{k} \sigma\left|H_{n r}\right| \Psi_{d}\right\rangle\left\langle\Psi_{d}\left|H_{e n}+H_{m a g n}\right| N, \vec{p} m_{s}, 0\right\rangle}{z-E_{d}^{0}-\Delta E_{d}+\frac{i}{2} \Gamma_{d}} .
\end{aligned}
$$

Here we introduce the notation $\Delta E_{d}=\Delta E_{d}^{\mathrm{NP}}+\Delta E_{d}^{\mathrm{SE}}+\Delta E_{d}^{\mathrm{NSE}}$ for the energy correction and $\Gamma_{d}=\Gamma_{d}^{\mathrm{IC}}+\Gamma_{d}^{\mathrm{n}, \mathrm{rad}}$ for the total natural width of the excited state $|d\rangle=\left|N^{*}, n_{d} \kappa_{d} m_{d}, 0\right\rangle$. 


\section{Differential and total cross sections for NEEC}

Equation (21) gives the differential cross section in terms of the matrix element of the projected $T$-operator. Neglecting the interference of neighboring resonances, and taking into account only a single state $d$, with the corresponding magnetic substates, the cross section for a given reaction pathway $i \rightarrow d \rightarrow f$ is

$$
\begin{aligned}
\frac{d \sigma_{i \rightarrow d \rightarrow f}}{d \Omega_{k}}(E) & =\frac{2 \pi}{F_{i}}\left|\left\langle N_{f} I_{f} M_{I_{f}}, n_{d} \kappa_{d} m_{d}, \vec{k} \sigma\left|H_{n r}\right| N_{d}^{*} I_{d} M_{I_{d}}, n_{d} \kappa_{d} m_{d}, 0\right\rangle\right|^{2} \\
& \times \frac{\left|\left\langle N_{d}^{*} I_{d} M_{I_{d}}, n_{d} \kappa_{d} m_{d}, 0\left|H_{e n}+H_{m a g n}\right| N_{i} I_{i} M_{I_{i}}, \overrightarrow{p m} m_{s}, 0\right\rangle\right|^{2}}{\left(E-E_{d}^{0}-\Delta E_{d}\right)^{2}+\frac{1}{4} \Gamma_{d}^{2}} \rho_{f} .
\end{aligned}
$$

$N_{i}$ as well as $N_{f}$ represent the nucleus in the ground state, while $N_{d}^{*}$ stands for the intermediate excited nuclear state. The angular momentum $I$ and its projection $M_{I}$ are used to denote the nuclear states. One has to perform an average over the magnetic substates of the initial state of the system and a summation over the final states if these are not resolved in a NEEC experiment. The total cross section is calculated by integrating over the solid angle $\Omega_{k}$ of the photon emission and averaging over the direction of the electron:

$$
\begin{aligned}
\sigma_{i \rightarrow d \rightarrow f}(E) & =\frac{2 \pi}{F_{i}} \sum_{M_{I_{f}} \sigma} \sum_{M_{I_{d}} m_{d}} \frac{1}{2\left(2 I_{i}+1\right)} \sum_{M_{I_{i}} m_{s}} \frac{1}{2 I_{d}+1} \sum_{M_{I_{d}}^{\prime}} \frac{1}{4 \pi} \int d \Omega_{p} \\
& \times \int d \Omega_{k} \frac{\left|\left\langle N_{f} I_{f} M_{I_{f}}, n_{d} \kappa_{d} m_{d}, \vec{k} \sigma\left|H_{n r}\right| N_{d}^{*} I_{d} M_{I_{d}}, n_{d} \kappa_{d} m_{d}, 0\right\rangle\right|^{2}}{\left(E-E_{d}\right)^{2}+\frac{1}{4} \Gamma_{d}^{2}} \\
& \times\left|\left\langle N_{d}^{*} I_{d} M_{I_{d}}, n_{d} \kappa_{d} m_{d}, 0\left|H_{e n}+H_{\text {magn }}\right| N_{i} I_{i} M_{I_{i}}, \overrightarrow{p m} m_{s}, 0\right\rangle\right|^{2} \rho_{f} .
\end{aligned}
$$

We denote the corrected energy of the intermediate state by $E_{d}=E_{d}^{0}+\Delta E_{d}$. By introducing the notation

$$
\begin{aligned}
Y_{n}^{i \rightarrow d} & =\frac{2 \pi}{2\left(2 I_{i}+1\right)} \sum_{M_{I_{i}} m_{s}} \sum_{M_{I_{d}} m_{d}} \\
& \times \int d \Omega_{p}\left|\left\langle N_{d}^{*} I_{d} M_{I_{d}}, n_{d} \kappa_{d} m_{d}, 0\left|H_{e n}+H_{m a g n}\right| N_{i} I_{i} M_{I_{i}}, \vec{p} m_{s}, 0\right\rangle\right|^{2} \rho_{i}
\end{aligned}
$$

for the electron capture rate,

$$
\begin{aligned}
A_{r}^{d \rightarrow f} & =\frac{2 \pi}{2 I_{d}+1} \sum_{M_{I_{f}} \sigma} \sum_{M_{I_{d}}} \\
& \times \int d \Omega_{k}\left|\left\langle N_{f} I_{f} M_{I_{f}}, n_{d} \kappa_{d} m_{d}, \vec{k} \sigma\left|H_{n r}\right| N_{d}^{*} I_{d} M_{I_{d}}, n_{d} \kappa_{d} m_{d}, 0\right\rangle\right|^{2} \rho_{f}
\end{aligned}
$$


for the radiative transition rate, and

$$
L_{d}\left(E-E_{d}\right)=\frac{\Gamma_{d} / 2 \pi}{\left(E-E_{d}\right)^{2}+\frac{1}{4} \Gamma_{d}^{2}}
$$

for the normalized Lorentz profile and taking into account the relation $F_{i} \rho_{i}=p^{2} /(2 \pi)^{3}$, the cross section formula can be written in the condensed form

$$
\sigma_{i \rightarrow d \rightarrow f}(E)=\frac{2 \pi^{2}}{p^{2}} \frac{A_{r}^{d \rightarrow f} Y_{n}^{i \rightarrow d}}{\Gamma_{d}} L_{d}\left(E-E_{d}\right) .
$$

Determining the total cross section of the studied process requires the calculation of the transition rates $Y_{n}$ and $A_{r}$, and the initial and final state energies. In the actual calculations we neglect the additional corrections $\Delta E_{d}^{\mathrm{NP}}$ and $\Delta E_{d}^{\mathrm{NSE}}$. The integration of the cross section over the continuum electron energy gives the resonance strength $S_{d}$ for a given recombined state $d$,

$$
S_{d}=\int d E \frac{2 \pi^{2}}{p^{2}} \frac{A_{r}^{d \rightarrow f} Y_{n}^{i \rightarrow d}}{\Gamma_{d}} L_{d}\left(E-E_{d}\right) .
$$

The natural width $\Gamma_{d}$ of the nuclear excited state is of the order of $10^{-6} \mathrm{eV}$. In this interval the values of $p^{2}$ as well as of the NEEC rate $Y_{n}$ can be considered constant. As the Lorentz profile is normalized to unity,

$$
\int d E L_{d}\left(E-E_{d}\right)=1
$$

the resonance strength can be written as

$$
S_{d}=\frac{2 \pi^{2}}{p^{2}} \frac{A_{r}^{d \rightarrow f} Y_{n}^{i \rightarrow d}}{\Gamma_{d}} .
$$

\section{RATES FOR ELECTRIC AND MAGNETIC TRANSITIONS}

In order to calculate the NEEC rate we have considered the matrix element of the electric and magnetic interactions between the electron and the nucleus. We write the wave function of the system as the product wave function of the electronic and nuclear states,

$$
H_{f i}=\left\langle N^{*} I_{d} M_{I_{d}}\left|\left\langle n_{d} \kappa_{d} m_{d}\left|H_{e n}+H_{m a g n}\right| \overrightarrow{p m} m_{s}\right\rangle\right| N I_{i} M_{I_{i}}\right\rangle .
$$

The initial state continuum electronic wave function is given through a partial wave expansion [16],

$$
\left|\vec{p} m_{s}\right\rangle=\sum_{\kappa m} i^{l} e^{i \Delta_{\kappa}} \sum_{m_{l}} Y_{l m_{l}}^{*}\left(\Omega_{e}\right) C\left(l \frac{1}{2} j ; m_{l} m_{s} m\right)|\varepsilon \kappa m\rangle
$$


where $\varepsilon$ is the energy of the continuum electron measured from the ionization threshold, $\varepsilon=\sqrt{p^{2} c^{2}+c^{4}}-c^{2}$. The orbital angular momentum of the partial wave is denoted by $l$ and the corresponding magnetic quantum number $m_{l}$ and the phases $\Delta_{\kappa}$ are chosen so that the continuum wave function fulfills the boundary conditions of an incoming plane wave and an outgoing spherical wave. The total angular momentum quantum number of the partial wave is $j=|\kappa|-\frac{1}{2}$. The $Y_{l m_{l}}\left(\Omega_{e}\right)$ denote the spherical harmonics and the $C\left(l \frac{1}{2} j ; m_{l} m_{s} m\right)$ stand for the vector coupling coefficients.

For describing the nucleus we have used a collective model [14] in which the excitations of the nucleus are assumed to be vibrations of the nuclear surface. The surface can be parametrized as

$$
R(\theta, \varphi, t)=R_{0}\left(1+\sum_{\lambda=0}^{\infty} \sum_{\mu=-\lambda}^{\lambda} \alpha_{\lambda \mu}^{*}(t) Y_{\lambda \mu}(\Omega)\right) .
$$

$R_{0}$ denotes the radius of a homogeneously charged sphere and the time-dependent amplitudes $\alpha_{\lambda \mu}$ serve as collective coordinates. Using this parametrization and requiring that the charge is homogeneously distributed, the nuclear charge density can be written as

$$
\rho_{n}(\vec{r}, t)=\rho_{0} \theta(R(\theta, \varphi, t)-r)
$$

with the constant average density $\rho_{0}=\frac{3 Z e}{4 \pi R_{0}^{3}}$. In the first order in the collective coordinates the density $\rho_{n}$ can be approximated as

$$
\rho_{n}(\vec{r}, t)=\rho_{0} \theta\left(R_{0}-r\right)+\rho_{0} R_{0} \delta\left(R_{0}-r\right) \sum_{\lambda \mu} \alpha_{\lambda \mu}^{*}(t) Y_{\lambda \mu}(\Omega)
$$

The electron-nucleus interaction Hamiltonian in eq. (13) can be written using the multipole expansion as

$$
H_{e n}=\sum_{L=0}^{\infty} \sum_{M=-L}^{L} \frac{4 \pi}{2 L+1} Y_{L M}^{*}\left(\Omega_{e}\right) \int d^{3} r \frac{r_{<}^{L}}{r_{>}^{L+1}} Y_{L M}\left(\Omega_{n}\right) \rho_{n}\left(\vec{r}_{n}\right) .
$$

The radius $r_{e}\left(r_{n}\right)$ denotes the electronic (nuclear) radial coordinate and $\Omega_{e}\left(\Omega_{n}\right)$ stands for the corresponding solid angle. We can make the simplifying assumption that the electron does not enter the nucleus, therefore we assume that $r_{e}>r_{n}$. According to Ref. [17], this approximation should not affect the results for the considered transitions. The Hamiltonian can then be written

$$
H_{e n}=\sum_{L=0}^{\infty} \sum_{M=-L}^{L} \frac{4 \pi}{2 L+1} Y_{L M}^{*}\left(\Omega_{e}\right) \frac{1}{r_{e}^{L+1}} Q_{L M}
$$


with the help of the electric multipole moments

$$
Q_{L M}=\int d^{3} r_{n} r_{n}^{L} Y_{L M}\left(\Omega_{n}\right) \rho_{n}\left(\vec{r}_{n}\right)
$$

After performing some angular momentum algebra one finds for the matrix element of $H_{e n}$ for a given partial wave component

$$
\begin{aligned}
& \left\langle N_{d}^{*} I_{d} M_{I_{d}}\left|\left\langle\kappa_{d} m_{d}\left|H_{e n}\right| \kappa m\right\rangle\right| N_{i} I_{i} M_{I_{i}}\right\rangle= \\
& \sum_{L M}(-1)^{I_{d}+M_{I_{i}}+L+M+m+3 j_{d}} R_{L, j_{d}, j}^{(1)}\left\langle N^{*} I_{d}\left\|Q_{L}\right\| N I_{i}\right\rangle \sqrt{2 j_{d}+1} \sqrt{\frac{4 \pi}{(2 L+1)^{3}}} \\
& \times C\left(I_{i} I_{d} L ;-M_{I_{i}} M_{I_{d}} M\right) C\left(j j_{d} L ;-m m_{d} M\right) C\left(j_{d} L j ; \frac{1}{2} 0 \frac{1}{2}\right) .
\end{aligned}
$$

$R_{L, j_{d}, j}^{(1)}$ stands for the radial integral given by

$$
R_{L, j_{d}, j}^{(1)}=\int_{0}^{\infty} d r r^{-L+1}\left(f_{\kappa_{d}}(r) f_{\varepsilon \kappa}(r)+g_{\kappa_{d}}(r) g_{\varepsilon \kappa}(r)\right)
$$

where $g(r)$ and $f(r)$ are the large and small radial components of the relativistic continuum electron wave function

$$
\Psi_{\varepsilon \kappa m}(\vec{r})=\left(\begin{array}{c}
g_{\varepsilon \kappa}(r) \Omega_{\kappa}^{m}\left(\Omega_{e}\right) \\
i f_{\varepsilon \kappa}(r) \Omega_{-\kappa}^{m}\left(\Omega_{e}\right)
\end{array}\right)
$$

and the $g_{d}(r)$ and $f_{d}(r)$ are the components of the bound Dirac wave functions

$$
\Psi_{n_{d} \kappa_{d} m_{d}}(\vec{r})=\left(\begin{array}{c}
g_{\kappa_{d}}(r) \Omega_{\kappa_{d}}^{m_{d}}\left(\Omega_{e}\right) \\
i f_{\kappa_{d}}(r) \Omega_{-\kappa_{d}}^{m_{d}}\left(\Omega_{e}\right)
\end{array}\right)
$$

with the spherical spinor functions $\Omega_{\kappa}^{m}$. For a given multipolarity $L$, the NEEC rate for an electric transition thus reads

$$
Y_{n}^{(e)}=\frac{4 \pi^{2} \rho_{i}}{(2 L+1)^{2}} B \uparrow\left(E L, I_{i} \rightarrow I_{d}\right)\left(2 j_{d}+1\right) \sum_{\kappa}\left|R_{L, j_{d}, j}^{(1)}\right|^{2} C\left(j_{d} L j ; \frac{1}{2} 0 \frac{1}{2}\right)^{2},
$$

where

$$
B \uparrow\left(E L, I_{i} \rightarrow I_{d}\right)=\frac{1}{2 I_{i}+1}\left|\left\langle N^{*} I_{d}\left\|Q_{L}\right\| N I_{i}\right\rangle\right|^{2}
$$

is the reduced electric transition probability.

If we consider the charge density of the nuclear collective model from eq. (56), the matrix element of the Hamiltonian $H_{e n}$ can be conveniently written in terms of the reduced 
transition probability $B \uparrow$ without imposing any constraints on the electron motion. In this case the electric NEEC rate is given by

$$
\begin{aligned}
Y_{n}^{(e)} & =\frac{4 \pi^{2} \rho_{i}}{(2 L+1)^{2}} R_{0}^{-2(L+1)} B \uparrow\left(E L, I_{i} \rightarrow I_{d}\right)\left(2 j_{d}+1\right) \\
& \times \sum_{\kappa}\left|R_{L, j_{d}, j}^{(2)}\right|^{2} C\left(j_{d} L j ; \frac{1}{2} 0 \frac{1}{2}\right)^{2}
\end{aligned}
$$

where the electronic radial integral is

$$
\begin{aligned}
R_{L, j_{d}, j}^{(2)} & =\frac{1}{R_{0}^{L-1}} \int_{0}^{R_{0}} d r r^{L+2}\left(f_{\kappa_{d}}(r) f_{\varepsilon \kappa}(r)+g_{\kappa_{d}}(r) g_{\varepsilon \kappa}(r)\right)+ \\
& +R_{0}^{L+2} \int_{R_{0}}^{\infty} d r r^{-L+1}\left(f_{\kappa_{d}}(r) f_{\varepsilon \kappa}(r)+g_{\kappa_{d}}(r) g(r)_{\varepsilon \kappa}\right)
\end{aligned}
$$

The magnetic Hamiltonian in eq. (27) can be written using the multipole expansion as

$$
H_{m a g n}=-\vec{\alpha} \cdot \vec{A}=-\frac{1}{c} \sum_{L M} \frac{4 \pi}{2 L+1} \vec{\alpha} \cdot \vec{Y}_{L L}^{M}\left(\Omega_{e}\right) \int d^{3} r_{n} \frac{r_{<}^{L}}{r_{>}^{L+1}} \vec{j}_{n}\left(\vec{r}_{n}\right) \cdot \vec{Y}_{L L}^{M *}\left(\Omega_{n}\right)
$$

We use again the approximation that the electron does not enter the nucleus. Then the Hamiltonian reads

$$
H_{\text {magn }}=-i \sum_{L M} \frac{4 \pi}{2 L+1} \sqrt{\frac{L+1}{L}} r_{e}^{-(L+1)} M_{L M} \vec{\alpha} \cdot \vec{Y}_{L L}^{M *}\left(\Omega_{e}\right),
$$

where the magnetic multipole operator is given by [18]

$$
M_{L M}=-\frac{i}{c} \sqrt{\frac{L}{L+1}} \int d^{3} r r^{L} \vec{Y}_{L L}^{M}\left(\Omega_{n}\right) \cdot \vec{j}_{n}\left(\vec{r}_{n}\right)
$$

Here, the vector spherical harmonics are defined as [19]

$$
\vec{Y}_{L L}^{M}\left(\Omega_{e}\right)=\sum_{\nu} \sum_{q} C(L 1 L ; \nu q M) Y_{L \nu}\left(\Omega_{e}\right) \vec{\epsilon}_{q}
$$

where $q=0, \pm 1$ and the spherical vectors $\vec{\epsilon}_{q}$ are

$$
\begin{aligned}
& \vec{\epsilon}_{+}=-\frac{1}{\sqrt{2}}\left(\vec{e}_{x}+i \vec{e}_{y}\right) \\
& \vec{\epsilon}_{0}=\vec{e}_{z} \\
& \vec{\epsilon}_{-}=\frac{1}{\sqrt{2}}\left(\vec{e}_{x}-i \vec{e}_{y}\right) .
\end{aligned}
$$


Introducing the expression for the vector spherical harmonics in eq. (69) we obtain

$$
\begin{aligned}
H_{\text {magn }} & =i \sum_{L M} \frac{4 \pi(-1)^{M}}{2 L+1} \sqrt{\frac{L+1}{L}} r^{-(L+1)} M_{L M} \\
& \times \sum_{\nu} Y_{L \nu}\left(\Omega_{e}\right)\left(-\frac{1}{\sqrt{2}} C(L 1 L ; \nu 1-M)\left(\alpha_{x}+i \alpha_{y}\right)\right. \\
& \left.+C(L 1 L ; \nu 0-M) \alpha_{z}+\frac{1}{\sqrt{2}} C(L 1 L ; \nu-1-M)\left(\alpha_{x}-i \alpha_{y}\right)\right),
\end{aligned}
$$

where $\alpha_{x}, \alpha_{y}$ and $\alpha_{z}$ are the Cartesian $\vec{\alpha}$ matrices. The matrix element of the magnetic Hamiltonian then yields

$$
\begin{aligned}
& \left\langle N^{*} I_{d} M_{I_{d}}\left|\left\langle n_{d} \kappa_{d} m_{d}|-\vec{\alpha} \cdot \vec{A}| \varepsilon \kappa m\right\rangle\right| N I_{i} M_{I_{i}}\right\rangle= \\
& 4 \pi i \sum_{L M \nu}(-1)^{M} \sqrt{\frac{L+1}{L}} \frac{1}{2 L+1}\left\langle N^{*} I_{d} M_{I_{d}}\left|M_{L M}\right| N I_{i} M_{I_{i}}\right\rangle \\
& \times\left(-\frac{1}{\sqrt{2}} C(L 1 L ; \nu 1-M)\left\langle n_{d} \kappa_{d} m_{d}\left|r^{-(L+1)} Y_{L \nu}\left(\Omega_{e}\right)\left(\alpha_{x}+i \alpha_{y}\right)\right| \varepsilon \kappa m\right\rangle\right. \\
& +C(L 1 L ; \nu 0-M)\left\langle n_{d} \kappa_{d} m_{d}\left|r^{-(L+1)} Y_{L \nu}\left(\Omega_{e}\right) \alpha_{z}\right| \varepsilon \kappa m\right\rangle \\
& \left.+\frac{1}{\sqrt{2}} C(L 1 L ; \nu-1-M)\left\langle n_{d} \kappa_{d} m_{d}\left|r^{-(L+1)} Y_{L \nu}\left(\Omega_{e}\right)\left(\alpha_{x}-i \alpha_{y}\right)\right| \varepsilon \kappa m\right\rangle\right) .
\end{aligned}
$$

Introducing the notations $T_{d i, \nu}^{+}, T_{d i, \nu}^{0}$ and $T_{d i, \nu}^{-}$for the electronic matrix elements we get

$$
\begin{aligned}
& \left\langle N^{*} I_{d} M_{I_{d}}\left|\left\langle n_{d} \kappa_{d} m_{d} \mid-\vec{\alpha} \cdot \overrightarrow{A \mid} \varepsilon \kappa m\right\rangle\right| N I_{i} M_{I_{i}}\right\rangle= \\
& 4 \pi i \sum_{L M \nu}(-1)^{I_{i}-M_{I_{i}}+M} \sqrt{\frac{L+1}{L(2 L+1)^{3}}} C\left(I_{d} I_{i} L ; M_{I_{d}}-M_{I_{i}} M\right)\left\langle N^{*} I_{d}|| M_{L}|| N I_{i}\right\rangle \\
& \times\left(-\frac{1}{\sqrt{2}} C(L 1 L ; \nu 1-M) T_{d i, \nu}^{+}+C(L 1 L ; \nu 0-M) T_{d i, \nu}^{0}\right. \\
& \left.+\frac{1}{\sqrt{2}} C(L 1 L ; \nu-1-M) T_{d i, \nu}^{-}\right) .
\end{aligned}
$$

The explicit form of the electronic matrix elements can be found in Appendix B, For a given multipolarity $L$, the rate for the nuclear excitation by electron capture for a pure magnetic transition is then

$$
\begin{aligned}
Y_{n}^{(m)} & =\frac{16 \pi^{3}(L+1) \rho_{i}}{L(2 L+1)^{3}} B \uparrow\left(M L, I_{i} \rightarrow I_{d}\right) \sum_{\kappa m m_{d}} \\
& \times \mid \sum_{M \nu}\left(-\frac{1}{\sqrt{2}} C(L 1 L ; \nu 1-M) T_{d i, \nu}^{+}+C(L 1 L ; \nu 0-M) T_{d i, \nu}^{0}\right. \\
& \left.+\frac{1}{\sqrt{2}} C(L 1 L ; \nu-1-M) T_{d i, \nu}^{-}\right)\left.\right|^{2} .
\end{aligned}
$$


All the nuclear information is contained in the reduced magnetic transition probability

$$
B \uparrow\left(M L, I_{i} \rightarrow I_{d}\right)=\frac{1}{2 I_{i}+1}\left|\left\langle N^{*} I_{d}\left\|M_{L}(t)\right\| N I_{i}\right\rangle\right|^{2},
$$

whose value can be taken from experimental data or from calculations involving different nuclear models. Given the different parity of the electric and magnetic multipole moments a transition of a given multipolarity $L$ is either electric or magnetic. We consider only the cases of transitions with a certain value of $L$ and we neglect the possible mixing ratios between electric and magnetic multipoles of different multipolarities.

\section{NUMERICAL RESULTS}

We calculate total cross sections and resonance strengths for NEEC followed by the radiative decay of the excited nucleus for various collision systems, involving electric $E 2$ and magnetic $M 1$ multipole transitions. We consider the cases of nuclear isotopes with low-lying nuclear levels for which the NEEC process is more likely to be observed experimentally.

For the case of electric multipole transitions we have considered the $0^{+} \rightarrow 2^{+} E 2$ transitions of the ${ }_{92}^{236} \mathrm{U},{ }_{92}^{238} \mathrm{U},{ }_{96}^{248} \mathrm{Cm},{ }_{70}^{174} \mathrm{Yb},{ }_{68}^{170} \mathrm{Er},{ }_{64}^{154} \mathrm{Gd},{ }_{64}^{156} \mathrm{Gd},{ }_{66}^{162} \mathrm{Dy}$ and ${ }_{66}^{164} \mathrm{Dy}$ even-even nuclei. The quadrupole excitations of even-even nuclei are well described by the collective model. For the calculation of the NEEC rate both formulas from eq. (64) and eq. (66) have been used for a comparison. A further $E 2$ transition $\frac{5}{2}^{-} \rightarrow \frac{7}{2}^{-}$of the odd ${ }_{66}^{163}$ Dy nucleus has been investigated. For this case we have calculated the NEEC rate assuming that the electron does not enter the nucleus. The reduced transition probability $B \uparrow(E 2)$ for the even-even nuclei as well as the energies of the nuclear levels were taken from [20], and in the case of the ${ }_{66}^{163} \mathrm{Dy}$ isotope, from [21]. The nuclear radiative rate was calculated according to the formula [22]

$$
A_{r}^{d \rightarrow f}(\lambda, L)=\frac{8 \pi(L+1)}{L((2 L+1) ! !)^{2}} \frac{E^{2 L+1}}{c} B \downarrow\left(\lambda L, I_{d} \rightarrow I_{f}\right),
$$

where $\lambda=E, M$ stands for the type of transition. The two reduced transition probabilities for the emission, respectively the absorption of a gamma ray are related through the formula

$$
B \downarrow\left(\lambda L, I_{d} \rightarrow I_{f}\right)=\frac{2 I_{f}+1}{2 I_{d}+1} B \uparrow\left(\lambda L, I_{f} \rightarrow I_{d}\right) .
$$

The width of the excited nuclear state is then

$$
\Gamma_{d}=A_{r}^{d \rightarrow f}+A_{\mathrm{IC}}^{d}
$$


where $A_{\mathrm{IC}}^{d}$ is the IC rate of the state $d$, related to the NEEC rate through the principle of detailed balance,

$$
A_{\mathrm{IC}}^{d}=\frac{2\left(2 I_{i}+1\right)}{\left(2 I_{d}+1\right)\left(2 j_{d}+1\right)} Y_{n}
$$

For the NEEC rate we need to calculate numerically the radial integrals $R_{L, j_{d}, j}$ that enter eq. (64) and eq. (66). We use relativistic Coulomb-Dirac wave functions for the continuum electron and wave functions calculated with the GRASP92 package [23] considering a homogeneously charged nucleus for the bound electron. The value of $R_{L, j_{d}, j}$ is almost the same whether we use Coulomb-Dirac radial wave functions or we take into account the finite size of the nucleus. The finite size of the nucleus has a sensitive effect on the energy levels of the bound electron. The energy level of the bound electron is calculated with GRASP92 and it includes quantum electrodynamic corrections. The first term of the sum of radial integrals in eq. (67) is about 3 orders of magnitude smaller than the second term,

$$
\begin{aligned}
& \frac{1}{R_{0}^{L-1}} \int_{0}^{R_{0}} d r r^{L+2}\left(f_{\kappa_{d}}(r) f_{\varepsilon \kappa}(r)+g_{\kappa_{d}}(r) g_{\varepsilon \kappa}(r)\right) \\
& \ll R_{0}^{L+2} \int_{R_{0}}^{\infty} d r r^{-L+1}\left(f_{\kappa_{d}}(r) f_{\varepsilon \kappa}(r)+g_{\kappa_{d}}(r) g_{\varepsilon \kappa}(r)\right) .
\end{aligned}
$$

Here, the nuclear radius $R_{0}$ is calculated according to the formula [24]

$$
R_{0}=\left(1.0793 A^{1 / 3}+0.73587\right) \mathrm{fm}
$$

where $A$ is the atomic mass number. If we make the approximation

$$
R_{L, j_{d}, j}^{(2)} \simeq R_{0}^{L+2} \int_{0}^{\infty} d r r^{-L+1}\left(f_{\kappa_{d}}(r) f_{\varepsilon \kappa}(r)+g_{\kappa_{d}}(r) g_{\varepsilon \kappa}(r)\right)
$$

the NEEC rate is exactly the one in (64), calculated with the assumption that the electron does not enter the nucleus. For the numerical cases of the even-even nuclei the difference between the rates calculated with eq. (64) and eq. (66) are from under $1 \%$ up to $6 \%$. The difference is larger for the capture into the $s$ orbitals and it increases with the value of the atomic number Z. For the capture of the continuum electron into the $2 s$ orbital of ${ }_{96}^{248} \mathrm{Cm}$, the value of the rate calculated using the non-penetrating approximation is $6 \%$ larger than the one calculated with the collective model, in which the restriction on the electron motion is avoided.

For the cases of the $\mathrm{U}$ isotopes and for ${ }_{96}^{248} \mathrm{Cm}$, the capture into the $\mathrm{K}$ shell is not possible due to the low energy level of the first excited nuclear state. For these 3 isotopes, 
TABLE I: Electric NEEC rates and resonance strengths for various heavy ion collision systems. $E_{\text {exc }}$ is the nuclear excitation energy, $E_{\mathrm{c}}$ is the continuum electron energy and $\Gamma_{\mathrm{d}}$ is the width of the excited nuclear state.

\begin{tabular}{|c|c|c|c|c|c|c|c|}
\hline Isotope & $E_{\text {exc }}(\mathrm{keV})$ & $E_{\mathrm{c}}(\mathrm{keV})$ & Type & Orbital & $Y_{\mathrm{n}}(1 / s)$ & $\Gamma_{\mathrm{d}}(\mathrm{eV})$ & $S($ barn $\cdot \mathrm{eV})$ \\
\hline${ }_{66}^{164} \mathrm{Dy}$ & 73.392 & 10.318 & E2 & $1 s_{1 / 2}$ & $1.86 \cdot 10^{8}$ & $4.36 \cdot 10^{-8}$ & $3.88 \cdot 10^{-2}$ \\
\hline${ }_{68}^{170} \mathrm{Er}$ & 78.591 & 11.350 & E2 & $1 s_{1 / 2}$ & $2.22 \cdot 10^{8}$ & $5.74 \cdot 10^{-8}$ & $4.70 \cdot 10^{-2}$ \\
\hline${ }_{70}^{174} \mathrm{Yb}$ & 76.471 & 4.897 & $\mathrm{E} 2$ & $1 s_{1 / 2}$ & $1.78 \cdot 10^{8}$ & $4.84 \cdot 10^{-8}$ & $9.25 \cdot 10^{-2}$ \\
\hline${ }_{64}^{154} \mathrm{Gd}$ & 123.071 & 64.005 & E2 & $1 s_{1 / 2}$ & $5.67 \cdot 10^{8}$ & $2.51 \cdot 10^{-7}$ & $2.90 \cdot 10^{-2}$ \\
\hline${ }_{64}^{156} \mathrm{Gd}$ & 88.966 & 74.742 & $\mathrm{E} 2$ & $2 s_{1 / 2}$ & $3.34 \cdot 10^{7}$ & $1.21 \cdot 10^{-7}$ & $7.07 \cdot 10^{-4}$ \\
\hline${ }_{64}^{156} \mathrm{Gd}$ & 88.966 & 74.896 & $\mathrm{E} 2$ & $2 p_{1 / 2}$ & $1.17 \cdot 10^{8}$ & $1.32 \cdot 10^{-7}$ & $2.26 \cdot 10^{-3}$ \\
\hline${ }_{64}^{156} \mathrm{Gd}$ & 88.966 & 75.680 & E2 & $2 p_{3 / 2}$ & $1.60 \cdot 10^{8}$ & $1.27 \cdot 10^{-7}$ & $3.17 \cdot 10^{-3}$ \\
\hline${ }_{66}^{162} \mathrm{Dy}$ & 80.660 & 65.432 & E2 & $2 s_{1 / 2}$ & $2.80 \cdot 10^{7}$ & $9.39 \cdot 10^{-8}$ & $6.23 \cdot 10^{-4}$ \\
\hline${ }_{66}^{162} \mathrm{Dy}$ & 80.660 & 66.594 & E2 & $2 p_{1 / 2}$ & $1.60 \cdot 10^{8}$ & $1.11 \cdot 10^{-7}$ & $2.99 \cdot 10^{-3}$ \\
\hline${ }_{66}^{162} \mathrm{Dy}$ & 80.660 & 66.492 & E2 & $2 p_{3 / 2}$ & $2.16 \cdot 10^{8}$ & $1.04 \cdot 10^{-7}$ & $4.25 \cdot 10^{-2}$ \\
\hline${ }_{66}^{163} \mathrm{Dy}$ & 73.440 & 58.212 & E2 & $2 s_{1 / 2}$ & $9.18 \cdot 10^{6}$ & $1.66 \cdot 10^{-7}$ & $1.33 \cdot 10^{-4}$ \\
\hline${ }_{66}^{163} \mathrm{Dy}$ & 73.440 & 58.374 & E2 & $2 p_{1 / 2}$ & $6.93 \cdot 10^{7}$ & $1.96 \cdot 10^{-7}$ & $8.54 \cdot 10^{-4}$ \\
\hline${ }_{66}^{163} \mathrm{Dy}$ & 73.440 & 58.272 & E2 & $2 p_{3 / 2}$ & $9.44 \cdot 10^{7}$ & $1.85 \cdot 10^{-7}$ & $1.21 \cdot 10^{-3}$ \\
\hline${ }_{92}^{236} \mathrm{U}$ & 45.242 & 11.113 & E2 & $2 s_{1 / 2}$ & $1.16 \cdot 10^{8}$ & $1.89 \cdot 10^{-8}$ & $8.79 \cdot 10^{-3}$ \\
\hline${ }_{92}^{236} \mathrm{U}$ & 45.242 & 11.038 & E2 & $2 p_{1 / 2}$ & $3.16 \cdot 10^{9}$ & $4.19 \cdot 10^{-7}$ & $1.05 \cdot 10^{-2}$ \\
\hline${ }_{92}^{236} \mathrm{U}$ & 45.242 & 15.601 & E2 & $2 p_{3 / 2}$ & $3.22 \cdot 10^{9}$ & $2.16 \cdot 10^{-7}$ & $1.56 \cdot 10^{-2}$ \\
\hline${ }_{92}^{238} \mathrm{U}$ & 44.910 & 10.782 & E2 & $2 s_{1 / 2}$ & $1.17 \cdot 10^{8}$ & $1.90 \cdot 10^{-8}$ & $8.90 \cdot 10^{-3}$ \\
\hline${ }_{92}^{238} \mathrm{U}$ & 44.910 & 10.706 & E2 & $2 p_{1 / 2}$ & $3.20 \cdot 10^{9}$ & $4.25 \cdot 10^{-7}$ & $1.06 \cdot 10^{-2}$ \\
\hline${ }_{92}^{238} \mathrm{U}$ & 44.910 & 15.269 & E2 & $2 p_{3 / 2}$ & $3.27 \cdot 10^{9}$ & $2.19 \cdot 10^{-7}$ & $1.56 \cdot 10^{-2}$ \\
\hline${ }_{96}^{248} \mathrm{Cm}$ & 43.380 & 5.500 & E2 & $2 s_{1 / 2}$ & $2.32 \cdot 10^{8}$ & $3.42 \cdot 10^{-8}$ & $1.79 \cdot 10^{-2}$ \\
\hline${ }_{96}^{248} \mathrm{Cm}$ & 43.380 & 5.398 & $\mathrm{E} 2$ & $2 p_{1 / 2}$ & $5.61 \cdot 10^{9}$ & $7.42 \cdot 10^{-7}$ & $1.91 \cdot 10^{-2}$ \\
\hline${ }_{96}^{248} \mathrm{Cm}$ & 43.380 & 11.018 & E2 & $2 p_{3 / 2}$ & $5.42 \cdot 10^{9}$ & $3.60 \cdot 10^{-7}$ & $2.20 \cdot 10^{-2}$ \\
\hline
\end{tabular}

recombination into the $\mathrm{L}$ shell of initially He-like ions is the most probable one. We regard the capture of the electron into a closed shell configuration as a one-electron problem, without the participation of the K-shell electrons. We consider for the continuum electron 
a total screening, while the bound electron is described by wave functions for an extended nucleus. The electron interaction is included in the bound radial wave functions calculated with GRASP92 and it influences the results through the value of the bound energy and through the shape of the electronic radial wave functions. The change of the shape of the radial wave functions has a larger numerical contribution to the value of the NEEC rate than the change of the energy due to the electron-electron interaction.

For the other cases of even-even nuclei, capture into the K shell is possible. For the ${ }_{64}^{156} \mathrm{Gd}$, ${ }_{66}^{162} \mathrm{Dy}$, and ${ }_{66}^{163} \mathrm{Dy}$ isotopes we have also considered the capture into the He-like ions. The width of the nuclear state in eq. (80) contains then an extra term which accounts for the possible IC of the K-shell electrons. The capture rate into the $2 p$ orbitals is in general one order of magnitude larger than the one for the capture into the $2 s$ orbital. The NEEC rates and resonance strengths for NEEC followed by the radiative decay of the nucleus for electric transitions are presented in Table 1 . The values of the NEEC rates have been calculated using the non-penetrating approximation.

For the magnetic multipole transitions we consider the $M 1$ transitions of the odd isotopes ${ }_{67}^{165} \mathrm{Ho},{ }_{70}^{173} \mathrm{Yb},{ }_{25}^{55} \mathrm{Mn},{ }_{26}^{57} \mathrm{Fe},{ }_{19}^{40} \mathrm{~K},{ }_{64}^{155} \mathrm{Gd},{ }_{64}^{157} \mathrm{Gd},{ }_{75}^{185} \mathrm{Re}$ and ${ }_{75}^{187} \mathrm{Re}$. Numerical results for these ions are presented in Table II. The electronic radial integrals are calculated numerically using the same type of wave functions for the bound and continuum electron as for the electric transitions. The reduced magnetic transition probability $B \downarrow(M 1)$ and the energies of the nuclear levels are taken from [25, 26, 27, 28, 29, 30, 31, 32, 33]. Capture into the K shell is possible for all the chosen ions, except for the ${ }_{64}^{157} \mathrm{Gd}$ isotope. Capture into the higher shells is less probable, and already for the capture into the $2 s$ orbital of ${ }_{67}^{167} \mathrm{Ho}$, the NEEC rate is one order of magnitude smaller. We present also results for captures into the He-like ions of ${ }_{64}^{155} \mathrm{Gd}$ and ${ }_{64}^{157} \mathrm{Gd}$. The largest resonance strength is the one for the capture into the $1 s$ orbital of ${ }_{70}^{173} \mathrm{Yb}$, namely, $S=8.57 \cdot 10^{-1} \mathrm{barn} \cdot \mathrm{eV}$. This value is small in comparison with the DR resonance strengths, which are in the order of magnitude of $10^{3}$ barn $\cdot \mathrm{eV}$.

In Figure 1 we present the cross section for the capture of the continuum electron into the $2 p_{1 / 2}$ and $2 p_{3 / 2}$ orbitals of the two studied even-even uranium isotopes. The cross sections for the capture into the $2 p_{3 / 2}$ orbitals are larger than the ones into the $2 p_{1 / 2}$ orbitals. Although the cross section values are in the order of thousands of barns, the width of the Lorentzian is given by the width of the excited nuclear state, which is in the order of $10^{-7} \mathrm{eV}$. This validates the use of the isolated resonance approximation in Section 【IC, 
TABLE II: Magnetic NEEC rates and resonance strengths for various heavy ion collision systems. $E_{\text {exc }}$ is the nuclear excitation energy, $E_{\mathrm{c}}$ is the continuum electron energy and $\Gamma_{\mathrm{d}}$ is the width of the excited nuclear state.

\begin{tabular}{lrrrrrrr}
\hline \hline Isotope & $E_{\text {exc }}(\mathrm{keV})$ & $E_{\mathrm{c}}(\mathrm{keV})$ & Type & Orbital & $Y_{\mathrm{n}}(1 / s)$ & $\Gamma_{\mathrm{d}}(\mathrm{eV})$ & $S(\mathrm{barn} \cdot \mathrm{eV})$ \\
\hline${ }_{67}^{165} \mathrm{Ho}$ & 94.700 & 29.563 & $\mathrm{M} 1$ & $1 s_{1 / 2}$ & $4.50 \cdot 10^{9}$ & $7.33 \cdot 10^{-6}$ & $4.95 \cdot 10^{-1}$ \\
${ }_{70}^{173} \mathrm{Yb}$ & 78.647 & 7.073 & $\mathrm{M} 1$ & $1 s_{1 / 2}$ & $2.52 \cdot 10^{9}$ & $2.43 \cdot 10^{-6}$ & $8.57 \cdot 10^{-1}$ \\
${ }_{75}^{185} \mathrm{Re}$ & 125.358 & 42.198 & $\mathrm{M} 1$ & $1 s_{1 / 2}$ & $9.20 \cdot 10^{9}$ & $1.51 \cdot 10^{-5}$ & $7.33 \cdot 10^{-1}$ \\
${ }_{75}^{187} \mathrm{Re}$ & 134.243 & 51.083 & $\mathrm{M} 1$ & $1 s_{1 / 2}$ & $8.82 \cdot 10^{9}$ & $1.67 \cdot 10^{-5}$ & $6.05 \cdot 10^{-1}$ \\
${ }_{25}^{55} \mathrm{Mn}$ & 125.949 & 117.378 & $\mathrm{M} 1$ & $1 s_{1 / 2}$ & $1.33 \cdot 10^{7}$ & $1.75 \cdot 10^{-6}$ & $5.02 \cdot 10^{-4}$ \\
${ }_{26}^{57} \mathrm{Fe}$ & 14.412 & 5.135 & $\mathrm{M} 1$ & $1 s_{1 / 2}$ & $2.24 \cdot 10^{6}$ & $1.25 \cdot 10^{-9}$ & $8.83 \cdot 10^{-4}$ \\
${ }_{19}^{40} \mathrm{~K}$ & 29.829 & 24.896 & $\mathrm{M} 1$ & $1 s_{1 / 2}$ & $6.03 \cdot 10^{6}$ & $8.85 \cdot 10^{-8}$ & $1.10 \cdot 10^{-3}$ \\
${ }_{64}^{155} \mathrm{Gd}$ & 60.008 & 45.784 & $\mathrm{M} 1$ & $2 s_{1 / 2}$ & $9.38 \cdot 10^{8}$ & $8.21 \cdot 10^{-7}$ & $2.62 \cdot 10^{-3}$ \\
${ }_{64}^{155} \mathrm{Gd}$ & 60.008 & 45.938 & $\mathrm{M} 1$ & $2 p_{1 / 2}$ & $1.04 \cdot 10^{7}$ & $7.84 \cdot 10^{-7}$ & $3.05 \cdot 10^{-4}$ \\
${ }_{64}^{155} \mathrm{Gd}$ & 60.008 & 46.722 & $\mathrm{M} 1$ & $2 p_{3 / 2}$ & $3.75 \cdot 10^{6}$ & $7.80 \cdot 10^{-7}$ & $1.08 \cdot 10^{-4}$ \\
${ }_{64}^{157} \mathrm{Gd}$ & 54.533 & 40.309 & $\mathrm{M} 1$ & $2 s_{1 / 2}$ & $1.42 \cdot 10^{8}$ & $3.17 \cdot 10^{-7}$ & $1.34 \cdot 10^{-2}$ \\
${ }_{64}^{157} \mathrm{Gd}$ & 54.533 & 40.463 & $\mathrm{M} 1$ & $2 p_{1 / 2}$ & $1.59 \cdot 10^{7}$ & $2.61 \cdot 10^{-7}$ & $1.82 \cdot 10^{-3}$ \\
${ }_{64}^{157} \mathrm{Gd}$ & 54.533 & 41.247 & $\mathrm{M} 1$ & $2 p_{3 / 2}$ & $5.81 \cdot 10^{6}$ & $2.56 \cdot 10^{-7}$ & $6.67 \cdot 10^{-4}$ \\
\hline \hline
\end{tabular}

\section{SUMMARY}

In this article we present a versatile formalism for describing complex processes actively involving atomic electrons and nuclei and derive a total cross section formula for the process of NEEC. The cross section can be traced back to the calculation of IC rates and radiative transition rates. It also requires the knowledge of nuclear excitation energies and the bound state energy of the electron after capture.

We derive NEEC rates for the case of both electric and magnetic multipole transitions using relativistic electronic wave functions. For the bound electron we use radial wave functions which take into account the finite size of the nucleus. The nucleus is described using a nuclear collective model. The nuclear part of the matrix element is written with the help of the reduced nuclear transition probability whose value is taken from experiment.

We calculate numerically the cross sections for NEEC followed by the radiative decay of 


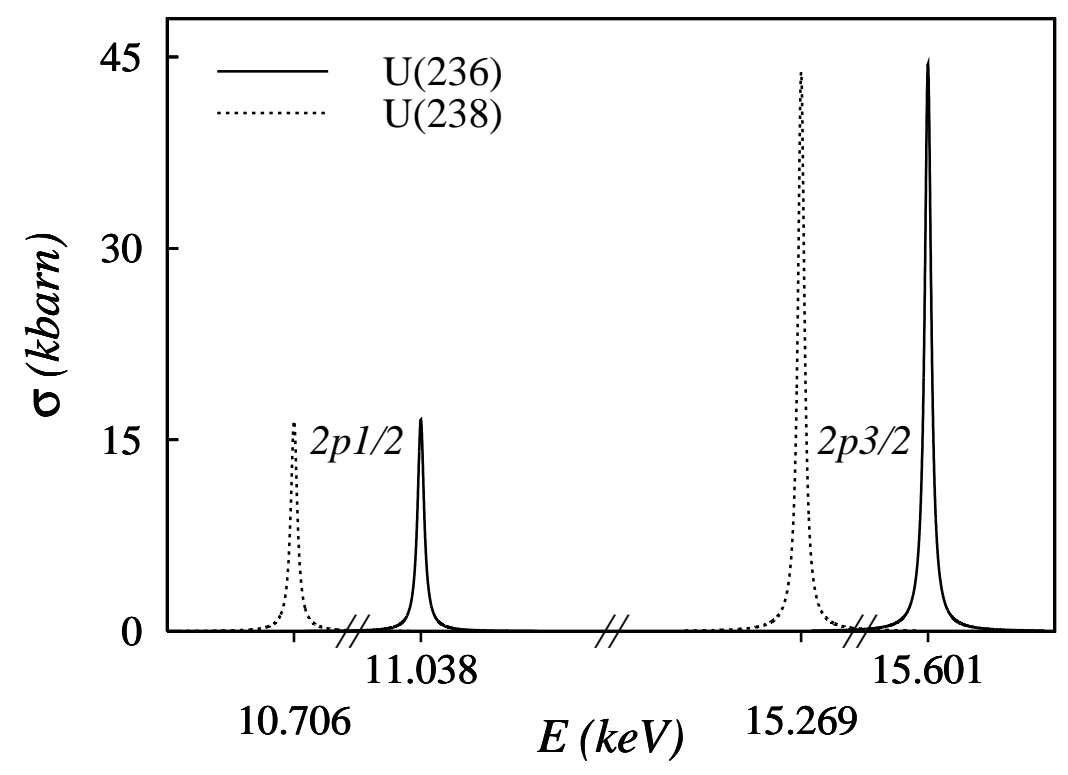

FIG. 1: NEEC cross sections for Uranium isotopes as a function of the continuum electron energy

the excited nuclear state for various nuclei. Particular interest is payed to the cases that are more likely to be observed experimentally. The obtained resonance strengths are typically 4 orders of magnitude smaller than the DR ones, which is due to the small width of the excited nuclear states.

If the initial and final states for NEEC are the same as the ones for the process of radiative recombination $(\mathrm{RR})$, quantum interference between the two processes occurs. The cross section for RR is much larger than the one of NEEC, therefore, the inclusion of interference terms is expected to increase the cross section by several orders of magnitude, making the experimental observation of NEEC feasible. Calculations about the magnitude of this effect are in progress.

\section{Acknowledgments}

The authors would like to thank Prof. R. V. Jolos for enlightening discussions regarding nuclear structure theory. Z. H. also appreciates useful discussions with Prof. H. Tawara and J. R. Crespo López-Urrutia about experimental issues of recombination processes. A. P. acknowledges the financial support from the Deutsche Forschungsgemeinschaft (DFG). 


\section{APPENDIX A:}

In this Appendix we show that the term $Q H_{n r} R G_{0} R H_{e r} P$ can be approximated by $Q H_{\text {magn }} P$, where we introduced the Hamiltonian

$$
H_{m a g n}=-\frac{1}{c} \vec{\alpha} \int d^{3} r_{n} \frac{\vec{j}_{n}\left(\vec{r}_{n}\right)}{\left|\vec{r}-\vec{r}_{n}\right|}
$$

describing the magnetic interaction of the nuclear and electric currents due to the exchange

of a transverse photon. The replacement is valid in the case when the frequency of the virtual photon is negligible, or, in other terms, when its wavelength is large compared to the typical linear size of the total system.

In this derivation we use the second quantized forms

$$
\begin{aligned}
H_{e r} & =\sum_{a b} \sum_{\sigma} \int d^{3} k \frac{1}{2 \pi} \sqrt{\frac{c}{k}} c_{a}^{\dagger} c_{b} \\
& \times \int d^{3} r_{e} \phi_{a}^{*}\left(\vec{r}_{e}\right) \vec{\alpha}\left(\vec{\epsilon}_{\vec{k} \sigma} a_{\vec{k} \sigma} e^{i \vec{k} \cdot \vec{r}_{e}}+\vec{\epsilon}_{\vec{k} \sigma}^{*} a_{\vec{k} \sigma}^{\dagger} e^{-i \vec{k} \cdot \vec{r}_{e}}\right) \phi_{b}\left(\vec{r}_{e}\right)
\end{aligned}
$$

and

$$
\begin{aligned}
H_{n r} & =-\frac{1}{(2 \pi)^{5 / 2}} \sqrt{\frac{1}{c k}} \sum_{s t} \sum_{\sigma^{\prime}} \int d^{3} k^{\prime} B_{s}^{\dagger} B_{t} \\
& \times \int d^{3} r_{n} \vec{j}_{n}\left(\vec{\epsilon}_{\vec{k}^{\prime} \sigma^{\prime}} a_{\vec{k}^{\prime} \sigma^{\prime}} e^{i \vec{k}^{\prime} \cdot \vec{r}_{n}}+\vec{\epsilon}_{\vec{k}^{\prime} \sigma^{\prime}}^{*} a_{\vec{k}^{\prime} \sigma^{\prime}}^{\dagger} e^{-i \vec{k}^{\prime} \cdot \vec{r}_{n}}\right)
\end{aligned}
$$

of the electromagnetic interaction Hamiltonians. The $\phi_{a}$ form a complete set of one-electron states, and the $c_{a}^{\dagger}\left(c_{b}\right)$ are electronic creation (annihilation) operators. The $\psi_{s}$ and $\psi_{t}$ denote nuclear states and the $B_{s}^{\dagger}$ and $B_{t}$ are the mode operators of the nuclear collective model like in (10). Here we only label these operators by one index for simplicity.

Substituting these operators into the matrix element of $Q H_{n r} R G_{0} R H_{e r} P$, we get

$$
\begin{aligned}
& \sum_{r} \frac{\left\langle q\left|H_{n r}\right| r\right\rangle\left\langle r\left|H_{e r}\right| \alpha \varepsilon\right\rangle}{z-E_{r}}=-\sum_{r} \sum_{a b s t} \sum_{\sigma \sigma^{\prime}} \int d^{3} k \int d^{3} k^{\prime} \frac{1}{(2 \pi)^{2}} \frac{1}{k} \\
& \times\left\langle q\left|B_{s}^{\dagger} B_{t} \int d^{3} r_{n} \vec{j}_{n} \cdot \vec{\epsilon}_{\vec{k}^{\prime} \sigma^{\prime}} a_{\vec{k}^{\prime} \sigma^{\prime}} e^{i \vec{k}^{\prime} \vec{r}_{n}}\right| r\right\rangle \\
& \times\left\langle r\left|c_{a}^{\dagger} c_{b} \int d^{3} r_{e} \phi_{a}^{*}\left(\vec{r}_{e}\right) \vec{\alpha} \cdot \vec{\epsilon}_{\vec{k} \sigma}^{*} a_{\vec{k} \sigma}^{\dagger} e^{-i \vec{k} \vec{r}_{e}} \phi_{b}\left(\vec{r}_{e}\right)\right| \alpha \varepsilon\right\rangle .
\end{aligned}
$$

Note that only the photon creation term of (A2) and the photon annihilation term of (A3) contribute. Introducing the notation

$$
M_{a b}^{e}(\vec{k}, \sigma)=\frac{1}{2 \pi} \sqrt{\frac{c}{k}} \int d^{3} r_{e} \phi_{a}^{*}\left(\vec{r}_{e}\right) \vec{\alpha} \cdot \vec{\epsilon}_{\vec{k} \sigma}^{*} e^{-i \vec{k} \vec{r}_{e}} \phi_{b}\left(\vec{r}_{e}\right)
$$




$$
M_{s t}^{n}\left(\vec{k}^{\prime}, \sigma^{\prime}\right)=-\frac{1}{2 \pi} \sqrt{\frac{1}{c k}} \int d^{3} r_{n} \vec{j}_{n} \cdot \vec{\epsilon}_{\vec{k}^{\prime} \sigma^{\prime}} e^{i \vec{k}^{\prime} \cdot \vec{r}_{n}}
$$

for the electronic and nuclear interaction matrix elements and taking into account $\vec{k}=\vec{k}^{\prime}$, $\sigma=\sigma^{\prime},(\underline{A 4})$ can be condensed as

$$
\begin{aligned}
& \sum_{r} \frac{\left\langle q\left|H_{n r}\right| r\right\rangle\left\langle r\left|H_{e r}\right| \alpha \varepsilon\right\rangle}{z-E_{r}}= \\
& \sum_{r} \sum_{a b s t} \sum_{\sigma} \int d^{3} k\left\langle q\left|B_{s}^{\dagger} B_{t} a_{\vec{k} \sigma}\right| r\right\rangle\left\langle r\left|c_{a}^{\dagger} c_{b} a_{\vec{k} \sigma}^{\dagger}\right| p\right\rangle \frac{M_{a b}^{e}(\vec{k}, \sigma) M_{s t}^{n}(\vec{k}, \sigma)}{z-E_{r}} .
\end{aligned}
$$

Evaluating the above expression at $z=\varepsilon_{b}^{e}+\varepsilon_{t}^{n}$, which is equal to the sum of the initial state electron and nuclear energies, only the $r$ state for which $E_{r}=\varepsilon_{a}^{e}+\varepsilon_{t}^{n}+c k$ holds has to be considered. Taking into account the property

$$
\sum_{\sigma}\left(\vec{a} \cdot \vec{\epsilon}_{\vec{k} \sigma}\right)\left(\vec{b} \cdot \vec{\epsilon}_{\vec{k} \sigma}\right)=\vec{a} \cdot \vec{b}-\frac{(\vec{a} \cdot \vec{k})(\vec{b} \cdot \vec{k})}{k^{2}}
$$

of the transversal polarization vectors that holds for any pair of vectors $\vec{a}$ and $\vec{b}$, we arrive to

$$
\sum_{r} \frac{\left\langle q\left|H_{n r}\right| r\right\rangle\left\langle r\left|H_{e r}\right| \alpha \varepsilon\right\rangle}{z-E_{r}}=\sum_{a b s t} \int d^{3} k\left\langle q\left|B_{s}^{\dagger} B_{t} c_{a}^{\dagger} c_{b}\right| p\right\rangle \frac{M_{a b s t}(\vec{k})}{\varepsilon_{b}^{e}-\varepsilon_{a}^{e}-c k} .
$$

$M_{a b s t}(\vec{k})$ denotes the product of electronic and nuclear matrix elements summed over the polarization directions

$$
\begin{aligned}
& M_{a b s t}(\vec{k})=\sum_{\sigma} M_{a b}^{e}(\vec{k}, \sigma) M_{s t}^{n}(\vec{k}, \sigma)=-\frac{1}{(2 \pi)^{2}} \frac{1}{k} \int d^{3} r_{e} \phi_{a}^{*}\left(\vec{r}_{e}\right) \\
& \times \int d^{3} r_{n} e^{i \vec{k} \cdot\left(\vec{r}_{n}-\vec{r}_{e}\right)}\left(\vec{j}_{n} \cdot \vec{\alpha}-\frac{\left(\overrightarrow{j_{n}} \cdot \vec{k}\right)(\vec{\alpha} \cdot \vec{k})}{k^{2}}\right) \phi_{b}\left(\vec{r}_{e}\right) .
\end{aligned}
$$

In the long wavelength limit $\vec{k} \rightarrow \overrightarrow{0}$, this further simplifies to

$$
M_{a b s t}(0)=-\frac{1}{(2 \pi)^{2}} \frac{1}{k} \int d^{3} r_{e} \phi_{a}^{*}\left(\vec{r}_{e}\right) \int d^{3} r_{n} \vec{j}_{n} \cdot \vec{\alpha} \phi_{b}\left(\vec{r}_{e}\right) .
$$

Applying the identity

$$
\frac{1}{w+i \epsilon}=\mathcal{P} \frac{1}{w}-i \pi \delta(w)
$$

where $\mathcal{P}$ implies the principal value integration, and adopting the long wavelength approximation, the real part of (A9) turns into

$$
\lim _{\epsilon \rightarrow 0} \frac{1}{2} \sum_{a b s t} \int d^{3} k\left\langle q\left|B_{s}^{\dagger} B_{t} c_{a}^{\dagger} c_{b}\right| \alpha \varepsilon\right\rangle M_{a b s t}(\vec{k})\left[\frac{1}{\varepsilon_{b}^{e}-\varepsilon_{a}^{e}-c k+i \epsilon}+\frac{1}{\varepsilon_{b}^{e}-\varepsilon_{a}^{e}-c k-i \epsilon}\right] .
$$


It can be rewritten as

$$
\sum_{a b s t}\left\langle q\left|B_{s}^{\dagger} B_{t} c_{a}^{\dagger} c_{b}\right| \alpha \varepsilon\right\rangle \int d^{3} r_{e} \phi_{a}^{*}\left(\vec{r}_{e}\right) V_{\text {magn }}\left(\vec{r}_{e}, \vec{r}_{n} ; \varepsilon_{b}^{e}-\varepsilon_{a}^{e}\right) \phi_{b}\left(\vec{r}_{e}\right)
$$

in terms of the effective magnetic potential

$$
\begin{aligned}
V_{\text {magn }}\left(\vec{r}_{e} ; \varepsilon_{b}^{e}-\varepsilon_{a}^{e}\right) & =-\frac{1}{2} \frac{1}{(2 \pi)^{2}} \frac{1}{k} \lim _{\epsilon \rightarrow 0} \int d^{3} k \int d^{3} r_{n}\left(\vec{j}_{n} \cdot \vec{\alpha}-\frac{\left(\vec{j}_{n} \cdot \vec{k}\right)(\vec{\alpha} \cdot \vec{k})}{k^{2}}\right) \\
& \times e^{i \vec{k} \cdot\left(\vec{r}_{n}-\vec{r}_{e}\right)}\left(\frac{1}{\varepsilon_{b}^{e}-\varepsilon_{a}^{e}-c k+i \epsilon}+\frac{1}{\varepsilon_{b}^{e}-\varepsilon_{a}^{e}-c k-i \epsilon}\right) \\
& =-\frac{1}{(2 \pi)^{2}} \frac{4 \pi}{\left|\vec{r}_{n}-\vec{r}_{e}\right|} \lim _{\epsilon \rightarrow 0} \int_{0}^{\infty} d k \int d^{3} r_{n}\left(\vec{j}_{n} \cdot \vec{\alpha}-\frac{\left(\overrightarrow{j_{n}} \cdot \vec{k}\right)(\vec{\alpha} \cdot \vec{k})}{k^{2}}\right) \\
& \times \sin \left(k\left|\vec{r}_{n}-\vec{r}_{e}\right|\right)\left(\frac{1}{\varepsilon_{b}^{e}-\varepsilon_{a}^{e}-c k+i \epsilon}+\frac{1}{\varepsilon_{b}^{e}-\varepsilon_{a}^{e}-c k-i \epsilon}\right)
\end{aligned}
$$

In the long wavelength limit, the $\vec{k}$-dependent part vanishes, and $V_{\text {magn }}\left(\vec{r}_{e} ; \varepsilon_{b}^{e}-\varepsilon_{a}^{e}\right)$ turns out to be

$$
V_{\text {magn }}\left(\vec{r}_{e} ; 0\right)=-\frac{1}{c} \vec{\alpha} \int d^{3} r_{n} \frac{\vec{j}_{n}\left(\vec{r}_{n}\right)}{\left|\vec{r}_{n}-\vec{r}_{e}\right|}
$$

This is equal to the magnetic Hamiltonian in (A1) Q.E.D. It can also be shown that the imaginary part associated with the Dirac delta term in (A11) vanishes if the frequency $\varepsilon_{a}^{e}-\varepsilon_{b}^{e}$ of the exchanged photon goes to zero.

\section{APPENDIX B:}

A further calculation of the three electronic matrix elements that enter, in eq. (74), the formula of the magnetic Hamiltonian, gives the following expressions for the first term $T_{d i, \nu}^{+}$:

$$
\begin{aligned}
& T_{d i, \nu}^{+}=-2 i(-1)^{m_{d}-\frac{1}{2}} \sqrt{\frac{(2 L+1)\left(2 l_{A}+1\right)\left(2 l_{B^{\prime}}+1\right)}{4 \pi}}\left(\begin{array}{ccc}
L & l_{A} & l_{B^{\prime}} \\
0 & 0 & 0
\end{array}\right)\left(\begin{array}{ccc}
L & l_{A} & l_{B^{\prime}} \\
\nu & m+\frac{1}{2} & \frac{1}{2}-m_{d}
\end{array}\right) \\
& \times C\left(l_{A} \frac{1}{2} j ; m+\frac{1}{2}-\frac{1}{2} m\right) C\left(l_{B^{\prime}} \frac{1}{2} j_{d} ; m_{d}-\frac{1}{2} \frac{1}{2} m_{d}\right) \int_{0}^{\infty} d r r^{-L+1} g_{\kappa}(r) f_{\kappa_{d}}(r) \\
& +2 i(-1)^{m_{d}-\frac{1}{2}} \sqrt{\frac{(2 L+1)\left(2 l_{A^{\prime}}+1\right)\left(2 l_{B}+1\right)}{4 \pi}}\left(\begin{array}{ccc}
L & l_{A^{\prime}} & l_{B} \\
0 & 0 & 0
\end{array}\right)\left(\begin{array}{ccc}
L & l_{A^{\prime}} & l_{B} \\
\nu & \frac{1}{2}-m_{d} m+\frac{1}{2}
\end{array}\right) \\
& \times C\left(l_{A^{\prime}} \frac{1}{2} j_{d} ; m_{d}-\frac{1}{2} \frac{1}{2} m_{d}\right) C\left(l_{B} \frac{1}{2} j ; m+\frac{1}{2}-\frac{1}{2} m\right) \int_{0}^{\infty} d r r^{-L+1} g_{\kappa_{d}}(r) f_{\kappa}(r),(\mathrm{B} 1)
\end{aligned}
$$


for the second term $T_{d i, \nu}^{0}$ :

$$
\begin{aligned}
& T_{d i, \nu}^{0}=-i(-1)^{m_{d}-\frac{1}{2}} \sqrt{\frac{(2 L+1)\left(2 l_{A}+1\right)\left(2 l_{B^{\prime}}+1\right)}{4 \pi}}\left(\begin{array}{ccc}
L & l_{A} & l_{B^{\prime}} \\
0 & 0 & 0
\end{array}\right)\left(\begin{array}{ccc}
L & l_{A} & l_{B^{\prime}} \\
\nu & m-\frac{1}{2} & \frac{1}{2}-m_{d}
\end{array}\right) \\
& \times C\left(l_{A} \frac{1}{2} j ; m-\frac{1}{2} \frac{1}{2} m\right) C\left(l_{B^{\prime}} \frac{1}{2} j_{d} ; m_{d}-\frac{1}{2} \frac{1}{2} m_{d}\right) \int_{0}^{\infty} d r r^{-L+1} f_{\kappa_{d}}(r) g_{\kappa}(r) \\
& +i(-1)^{m_{d}+\frac{1}{2}} \sqrt{\frac{(2 L+1)\left(2 l_{A}+1\right)\left(2 l_{B^{\prime}}+1\right)}{4 \pi}}\left(\begin{array}{ccc}
L & l_{A} & l_{B^{\prime}} \\
0 & 0 & 0
\end{array}\right)\left(\begin{array}{ccc}
L & l_{A} & l_{B^{\prime}} \\
\nu & m+\frac{1}{2} & -\frac{1}{2}-m_{d}
\end{array}\right) \\
& \times C\left(l_{A} \frac{1}{2} j ; m+\frac{1}{2}-\frac{1}{2} m\right) C\left(l_{B^{\prime}} \frac{1}{2} j_{d} ; m_{d}+\frac{1}{2}-\frac{1}{2} m_{d}\right) \int_{0}^{\infty} d r r^{-L+1} f_{\kappa_{d}}(r) g_{\kappa}(r) \\
& +i(-1)^{m_{d}-\frac{1}{2}} \sqrt{\frac{(2 L+1)\left(2 l_{A^{\prime}}+1\right)\left(2 l_{B}+1\right)}{4 \pi}}\left(\begin{array}{ccc}
L & l_{A^{\prime}} & l_{B} \\
0 & 0 & 0
\end{array}\right)\left(\begin{array}{ccc}
L & l_{A^{\prime}} & l_{B} \\
\nu & -\frac{1}{2}-m_{d} & m+\frac{1}{2}
\end{array}\right) \\
& \times C\left(l_{A^{\prime}} \frac{1}{2} j_{d} ; m_{d}+\frac{1}{2}-\frac{1}{2} m_{d}\right) C\left(l_{B} \frac{1}{2} j ; m+\frac{1}{2}-\frac{1}{2} m\right) \int_{0}^{\infty} d r r^{-L+1} f_{\kappa}(r) g_{\kappa_{d}}(r) \\
& +i(-1)^{m_{d}-\frac{1}{2}} \sqrt{\frac{(2 L+1)\left(2 l_{A^{\prime}}+1\right)\left(2 l_{B}+1\right)}{4 \pi}}\left(\begin{array}{ccc}
L & l_{A^{\prime}} & l_{B} \\
0 & 0 & 0
\end{array}\right)\left(\begin{array}{ccc}
L & l_{A^{\prime}} & l_{B} \\
\nu & \frac{1}{2}-m_{d} & m-\frac{1}{2}
\end{array}\right) \\
& \times C\left(l_{A^{\prime}} \frac{1}{2} j_{d} ; m_{d}-\frac{1}{2} \frac{1}{2} m_{d}\right) C\left(l_{B} \frac{1}{2} j ; m-\frac{1}{2} \frac{1}{2} m\right) \int_{0}^{\infty} d r r^{-L+1} f_{\kappa}(r) g_{\kappa_{d}}(r),
\end{aligned}
$$

and for the third term $T_{d i, \nu}^{-}$:

$$
\begin{aligned}
& T_{d i, \nu}^{-}=-2 i(-1)^{m_{d}+\frac{1}{2}} \sqrt{\frac{(2 L+1)\left(2 l_{A}+1\right)\left(2 l_{B^{\prime}}+1\right)}{4 \pi}}\left(\begin{array}{ccc}
L & l_{A} & l_{B^{\prime}} \\
0 & 0 & 0
\end{array}\right)\left(\begin{array}{ccc}
L & l_{A} & l_{B^{\prime}} \\
\nu & m-\frac{1}{2} & -m_{d}-\frac{1}{2}
\end{array}\right) \\
& \times C\left(l_{A} \frac{1}{2} j ; m-\frac{1}{2} \frac{1}{2} m\right) C\left(l_{B^{\prime}} \frac{1}{2} j_{d} ; m_{d}+\frac{1}{2}-\frac{1}{2} m_{d}\right) \int_{0}^{\infty} d r r^{-L+1} f_{\kappa_{d}}(r) g_{\kappa}(r) \\
& +2 i(-1)^{m_{d}+\frac{1}{2}} \sqrt{\frac{(2 L+1)\left(2 l_{A^{\prime}}+1\right)\left(2 l_{B}+1\right)}{4 \pi}}\left(\begin{array}{ccc}
L & l_{A^{\prime}} & l_{B} \\
0 & 0 & 0
\end{array}\right)\left(\begin{array}{ccc}
L & l_{A^{\prime}} & l_{B} \\
\nu & -m_{d}-\frac{1}{2} & m-\frac{1}{2}
\end{array}\right) \\
& \times C\left(l_{A^{\prime}} \frac{1}{2} j_{d} ; m_{d}+\frac{1}{2}-\frac{1}{2} m_{d}\right) C\left(l_{B} \frac{1}{2} j ; m-\frac{1}{2} \frac{1}{2} m\right) \int_{0}^{\infty} d r r^{-L+1} g_{\kappa_{d}}(r) f_{\kappa}(r) .
\end{aligned}
$$

Here $l_{A}$ and $l_{B}$ are the orbital quantum numbers for the upper and lower two component spinors of the initial continuum wave function. For the wave function of the final bound state the prime indices $l_{A^{\prime}}$ and $l_{B^{\prime}}$ are used. For a given value $\kappa$, the following relations hold:

$$
\begin{gathered}
j=|\kappa|-\frac{1}{2}, \\
l_{A}=\left\{\begin{array}{c}
\kappa \quad \text { if } \kappa>0, \\
|\kappa|-1 \text { if } \kappa<0,
\end{array}\right.
\end{gathered}
$$




$$
l_{B}=\left\{\begin{array}{c}
\kappa-1 \text { if } \kappa>0 \\
|\kappa| \quad \text { if } \kappa<0
\end{array}\right.
$$

[1] V. Goldanskii and V. A. Namiot, Phys. Lett. 62B, 393 (1976).

[2] M. Harston and J. Chemin, Phys. Rev. C 59, 2462 (1999).

[3] N. Cue, J.-C. Poizat, and J. Remillieux, Eurphys. Lett. 8, 19 (1989).

[4] J. Kimball, D. Bittle, and N. Cue, Phys. Lett. 152, 367 (1991).

[5] Z.-S. Yuan and J. Kimball, Phys. Rev. C 47, 323 (1993).

[6] I. Band and M. Trzhaskovskaya, At. Dat. Nucl. Dat. Tabl. 55, 43 (1993).

[7] S. Kishimoto, Y. Yoda, M. Seto, Y. Kobayashi, S. Kitao, R. Haruki, T. Kawauchi, K. Fukutani, and T. Okano, Phys. Rev. Lett. 85, 1831 (2000).

[8] T. Carreyre, M. R. Harston, M. Aiche, F. Bourgine, J. F. Chemin, G. Claverie, J. P. Goudour, J. N. Scheurer, F. Attallah, G. Bogaert, et al., Phys. Rev. C 62, 024311 (2000).

[9] A. Wolf, G. Gwinner, J. Linkemann, A. Saghiri, M. Schmitt, D. Schwalm, M. Grieser, M. Beutelspacher, T. Bartsch, C. Brandau, et al., Nucl. Instrum. Meth. Phys. Res. A 441, 183 (2000).

[10] A. Müller and S. Schippers, ASP Conf. Series 247, 53 (2001).

[11] A. J. G. Martínez, J. R. C. López-Urrutia, J. Braun, G. Brenner, H. Bruhns, A. Lapierre, V. Mironov, R. S. Orts, H. Tawara, M. Trinczek, et al., Phys. Rev. Lett. 94, 203201 (2005).

[12] D. Knapp, Z. Phys. D 21, 143 (1991).

[13] M. Zimmermann, N. Grün, and W. Scheid, J. Phys. B 30, 5259 (1997).

[14] W. Greiner and J. Maruhn, Nuclear Models (Springer Verlag Berlin Heidelberg, 1996).

[15] S. Zakowicz, W. Scheid, and N. Grün, J. Phys. B 37, 131 (2004).

[16] J. Eichler and W. Meyerhof, Relativistic Atomic Collisions (Academic Press San Diego, 1995).

[17] T. Green and M. Rose, Phys. Rev. 110, 105 (1958).

[18] C. Schwartz, Phys. Rev. 97, 380 (1955).

[19] A. R. Edmonds, Angular Momentum in Quantum Mechanics (Princeton University Press, 1996).

[20] S. Raman, C. Nestor, and P. Tikkanen, At. Dat. Nucl. Dat. Tabl. 78, 1 (2001).

[21] T. Burrows, Nucl. Dat. Sheets 56, 313 (1989).

[22] P. Ring and P. Schuck, The Nuclear Many-Body Problem (Springer Verlag New York, 1980). 
[23] F. A. Parpia, C. Froese-Fischer, and I. P. Grant, Comp. Phys. Comm. 94, 249 (1996).

[24] W. R. Johnson and G. Soff, At. Dat. Nucl. Dat. Tabl. 33, 405 (1985).

[25] R. B. Firestone, Nucl. Dat. Sheets 62, 159 (1991).

[26] R. G. Hehner, Nucl. Dat. Sheets 55, 71 (1988).

[27] V. S. Shirley, Nucl. Dat. Sheets 54, 589 (1988).

[28] L. K. Peker, Nucl. Dat. Sheets 65, 439 (1992).

[29] E. Browne, Nucl. Dat. Sheets 74, 165 (1995).

[30] C. W. Reich, Nucl. Dat. Sheets 71, 709 (1994).

[31] J. Huo, Nucl. Dat. Sheets 64, 723 (1991).

[32] M. R. Bhat, Nucl. Dat. Sheets 67, 195 (1992).

[33] J. A. Cameron and B. Singh, Nucl. Dat. Sheets 102, 293 (2004). 